\title{
Annotations
}

\section{Screening for congenital hypothyroidism}

In 1977 one of us (DBG) was co-author of an annotation in the Archives on early detection of congenital hypothyroidism. ${ }^{1}$ At that time it was already clear that screening of the newborn was generally feasible although there was still controversy about the most effective method. While it was generally accepted that the outlook for intelligence was better in hypothyroid children who started treatment soon after birth, there was almost no direct evidence on the value of screening in preventing later neurological and psychological handicap. We wish to reconsider these points in the light of recent experience.

During the last 4 years population screening for congenital hypothyroidism has gained momentum in Europe, North America, Australia, and Japan. Most European countries have introduced regional or national screening programmes and by 1979 the Newborn Committee of the European Thyroid Association was able to report the results in nearly half a million infants. ${ }^{2}$ In this country the DHSS is still considering the question of hypothyroid screening, but may soon recommend its introduction and funding on a regional basis, a decision which has already been taken in several parts of the country as a research or service commitment. The administrative and financial advantages of using the specimens already collected for phenylketonuria screening are considerable but in some areas the addition of hypothyroid screening would require reorganisation of the existing phenylketonuria programmes on to a regional basis because of the capital cost of the equipment necessary for radioimmunoassay.

There is now general agreement that screening by thyroxine (T4) assay alone results in lower detection rates and a higher incidence of borderline and falsepositive results. Thyrotrophin (TSH) assay cannot identify rare cases of secondary hypothyroidism but has the great advantage of a very low recall rate for repeat tests (about 1:1000) with few borderline values and no false-positives due to thyroxinebinding globulin deficiency. The choice thus lies between using TSH assay alone, or a primary T4 assay with TSH determination on specimens with low T4 values. Europe and Japan have generally chosen the former while many of the North American programmes use the combined method. In the UK both methods are being used and it is clear that provided relatively high cut-off $\mathrm{T} 4$ values are used for subsequent TSH assay ${ }^{3}$ most cases will be diagnosed by either method. However, it is unlikely that the risk of laboratory or clerical errors can ever be eliminated entirely, and in suspicious cases the diagnosis should not be discounted because the screening results were negative. In positive cases, the diagnosis must be confirmed by repeat tests (serum T4 and TSH, $x$-ray of knee for bone age, etc.) but this should not be allowed to delay treatment; if symptoms suggesting hypothyroidism are already present, treatment can be started as soon as the appropriate blood samples have been taken. Temporary withdrawal of treatment at a later date will clarify the situation in borderline cases, and will also identify those with transient hypothyroidism.

Some interesting aspects of congenital hypothyroidism have come to light as a result of population screening. The incidence in north London $(1: 3360)^{4}$ is close to that found in other TSH screening programmes, ${ }^{5}$ and is considerably higher than that in retrospective studies from Holland $(1: 6300),{ }^{6}$ and Sweden $(1: 6900){ }^{7}$ This discrepancy may not be fully explained by mild cases presenting later as 'juvenile hypothyroidism' and it is possible that some children with persistent increases in TSH previously escaped detection. When assessing the results of screening programmes, allowance must be made for these mild cases with large ectopic glands or partial defects of thyroxine biosynthesis who may have done well even if treatment had been long delayed.

It has also become apparent that many sick premature infants have depressed serum T4 levels, sometimes associated with transient increase in TSH. ${ }^{8}$ It is not yet clear whether this should be regarded as a pathological state requiring treatment or as a physiological adaptation to stress. We have encountered transient and unexplained increases in TSH in a few apparently healthy term babies, and transient neonatal hypothyroidism has also been described with excessive iodine uptake after fetography or even through the skin. ${ }^{9}$

Our experience with screening has convinced us that the clinical features of congenital hypothyroidism may be very difficult to recognise in the first 
month of life. While about three-quarters of our patients had symptoms - such as, feeding difficulties, lethargy, constipation, or prolonged jaundice-often these were not recognised as being abnormal, even by the parents. In only $2(6 \%)$ out of 36 cases was the diagnosis made before the screening results became available. We do not believe that increased awareness of the early symptoms of hypothyroidism with selective investigation of suspected cases can provide an effective alternative to population screening.

The preliminary results of follow-up in cases detected by screening are very encouraging: 20 infants diagnosed in Quebec had normal psychological and neuromuscular development on the Griffiths's scale at 18 months of age. ${ }^{5}$ In our own series, 15 out of 17 patients reviewed at one year had normal Griffiths's quotients (mean 102 \pm 8 ): the two exceptions were a child who also had cerebral gigantism, and a child born to a mother of subnormal intelligence. Whether the majority of these children will escape significant neurological or intellectual deficits in later life remains to be seen, particularly as there is evidence that some hypothyroid children treated before age 6 weeks have signs of 'minimal brain dysfunction' at later assessment. ${ }^{10-11}$ Continued careful assessment of cases both at the time of diagnosis and in later childhood will be essential in order to evaluate the benefits of early treatment and to determine whether even 1 or 2 weeks' delay in starting treatment has a significant effect on prognosis. There is a strong case for coordinating these initial and follow-up studies on a regional or national basis, perhaps in conjunction with the existing programme for phenylketonuria.

While the vital question concerning the value of screening in improving long-term prognosis cannot be fully answered until sufficient index cases have been followed for a number of years, the present situation gives no grounds for complacency. One of of us (JAH) has recently completed a survey of children with congenital hypothyroidism born between 1963 and 1977 in London and the surrounding area. Of the 112 children of school age, $28(25 \%)$ had IQs below 70 , and $32(29 \%)$ were attending special schools; a further 23 of those at normal schools required remedial teaching. In those treated before age 1 year there was an inverse relationship between IQ and the age of starting treatment: the 6 children treated before 6 weeks all had a normal IQ and attended normal schools. There was no significant improvement in the mean age of diagnosis between 1963 and 1977, and only $42(32 \%)$ of the 132 children had been diagnosed by 3 months of age. These results, which are similar to those in previous studies, ${ }^{12-13}$ add further weight to the argument that widespread screening should be introduced without delay.

\section{References}

1 MacFaul R, Grant D B. Early detection of hypothyroidism. Arch Dis Child 1977; 52: 87-8.

2 Delange F. Neonatal screening for congenital hypothyroidism in Europe. Report of the Newborn Committee of the European Thyroid Association. Acta Endocrinol [Suppl] (Kbh) 1979; 90: Supplement 223, 3-29.

3 Morissettee J, Dussault J H. Commentary: The cut-off point for TSH measurement or recalls in a screening program for congenital hypothyroidism using primary $\mathrm{T} 4$ screening. J Pediatr 1979; 95: 404-6.

- Hulse J A, Grant D B, Clayton B E, et al. Population screening for congenital hypothyroidism. Br Med J 1980; 280: 675-8.

5 Fisher D A, Dussault J H, Foley T P R, et al. Screening for congenital hypothyroidism: results of screening one million North American infants. J Pediatr 1979; 94: 700-5.

- de Jonge C A. Letter: Congenital hypothyroidism in the Netherlands. Lancet 1976; ii: 143.

7 Alm J, Larssen A, Zetterstrom R. Congenital hypothyroidism in Sweden. Acta Paediatr Scand 1978; 67: 1-3.

8 Schonberger W, Grimm W, Gerupp W, Dinkel E. Transient hypothyroidism associated with prematurity, sepsis, and respiratory distress. Eur J Pediatr 1979; 132: 85-92.

- Brown R S, Braverman L E. Transient hypothyroidism in a premature infant after skin preparation with iodine (abstract). Pediatr Res 1980; 14 : 475.

10 Wolter R, Noel P, De Lock P, et al. Neurophysiological study in treated thyroid dysgenesis. Acta Paediatr Scand (Suppl) 1979; Supplement 277, 41-6.

11 MacFaul R, Dorner S, Brett E M, Grant D B. Neurological abnormalities in patients treated for hypothyroidism from early life. Arch Dis Child 1978; 53: 611-9.

12 Raiti S, Newns G H. Cretinism: early diagnosis and its relation to mental prognosis. Arch Dis Child 1971; 46: $692-4$.

13 Klein A H, Meltzer S, Kenny F M. Improved prognosis of congenital hypothyroidism treated before age three months. J Pediatr 1972; 81 : 912-5.

D B Grant AND J A Hulse The Hospital for Sick Children, Great Ormond Street, London WC1N $3 J H$ 